\title{
AST/RO AND SUBMILLIMETRE-WAVE OBSERVATIONS OF THE MAGELLANIC CLOUDS FROM THE SOUTH POLE
}

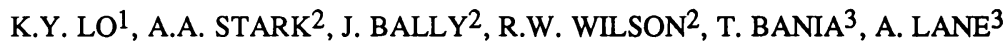 \\ 1 Astronomy Department, University of Illinois, $1101 \mathrm{~W}$. Springfield Ave, Urbana IL \\ 61801, USA \\ 2 AT\&T Bell Laboratories, 600 Mountain Ave, Murray Hill NJ 07974, USA \\ 3 Astronomy Department, Boston University, 147 Bay State Road, Boston MA 02215, USA
}

\begin{abstract}
AST/RO (Antarctic Submillimeter Telescope and Remote Observatory), a joint project between AT\&T Bell Labs, Boston University and the University of Illinois, will place a $1.7-\mathrm{m}$ submillimetre telescope at the South Pole (a high, dry site) in 1992. The highly automated off-axis parabolic telescope will be equipped initially with two channels of $500 \mathrm{GHz}(600 \mu \mathrm{m})$ heterodyne receivers and AOS spectrometers. A primary goal of the project is a large-scale survey of $\mathrm{CI}$ and $\mathrm{CO}(4-3)$ line emission from the southern Galaxy and the Magellanic Clouds, in an effort to further the understanding of the warm interstellar medium and star formation in the two galaxies.
\end{abstract}

\section{Introduction}

The bulk of the thermal radiation from the warm interstellar medium, near young massive stars and other sources of excitation, falls in the submillimetre wavelength range, $1 \mu \mathrm{m}>\lambda>100 \mu \mathrm{m}$. This wavelength range is also very rich spectroscopically, containing the higher rotational transitions of molecules, and atomic fine structure lines of the abundant elements. Thus, the submillimetre wavelength range is particularly suitable for probing the conditions of star formation and galaxy formation.

One of the major limitations of ground-based submillimetre-wave observations is the opacity of the atmosphere. At the best northern hemisphere site, Mauna Kea in Hawaii, submillimetre-wave observations in the $350 \mu \mathrm{m}$ and $650 \mu \mathrm{m}$ windows are possible only during the best conditions, about $30 \%$ of the time. Because of its lack of precipitation, low temperature and high altitude, the interior of Antarctica offers the best site for ground-based submillimetre observations in terms of atmospheric transmission. Measurements from the South Pole reported by Pajot $e$ t al. (1989) and Dragovan et al. (1990) indicate that the broad-band atmospheric opacity in the $650 \mu \mathrm{m}$ window is typically $\tau_{\mathrm{z}} \sim 0.2$ in the summer. In comparison, the best conditions at Mauna Kea correspond to $\tau_{\mathrm{z}} \sim 1$ in the same band. In the Arctic winter, conditions at one millimetre wavelength are likely to be essentially as good as the Kuiper Airborne Observatory. Thus, submillimetre-wave observations at the South Pole should be routine. 


\section{The AST/RO Telescope}

AST/RO is a 1.7-m diameter submillimetre-wave telescope, funded by the US National Science Foundation, scheduled for permanent installation at the South Pole in late 1992. The offset paraboloid primary is made of carbon fibre and epoxy, with a vacuum deposited aluminium surface with a surface roughness of $6 \mu \mathrm{m}$, and an rms figure of about $14 \mu \mathrm{m}$. The telescope is intended for diffraction limited operation down to the $350 \mu \mathrm{m}$ window, with a beam-size of $96 "(\lambda=600$ $\mu \mathrm{m})$. The Gregorian secondary is a prolate spheroid. Diffraction limited field of view is $3^{\prime}$ in diameter at $\lambda=\sim 3 \mu \mathrm{m}$ and 30' in diameter at $\lambda=200 \mu \mathrm{m}$. The telescope has a Naismith focus, with the beam passing through an elevation bearing with a $0.3 \mathrm{~m}$ diameter hole. The receivers will be in a heated Coudé room under the mount. To ensure the reliability of the system, there will be a two-fold redundancy of the receiver components, cryogenic systems, and the spectrometers. The receivers will be based on Niobium SIS mixers, operating at $4 \mathrm{~K}$. The spectrometers are acoustooptic spectrometers (AOS), with $1 \mathrm{GHz}$ bandwidth and 2000 channels, being built by the Kosma group at the University of Cologne (Schieder et al. 1989). The system will be highly automated to reduce to a minimum the need for operator intervention.

\section{AST/RO and the Magellanic Clouds}

The cold molecular phase of the interstellar medium in the Magellanic Clouds has been studied only recently with the CO(1-0) survey by Cohen et al. (1988) and by observations with SEST (Booth \& Johansson 1990). With AST/RO, the regions with detected CO will be surveyed for the $609 \mu \mathrm{m}$ CI fine structure line and the $650 \mu \mathrm{m} \mathrm{CO} \mathrm{(4-3)} \mathrm{line,} \mathrm{providing} \mathrm{probes} \mathrm{of} \mathrm{the} \mathrm{warm} \mathrm{interstellar}$ medium. Given the different stellar populations and elemental abundances in the Magellanic Clouds compared to the Galaxy, the conditions of the interstellar medium in the Magellanic Clouds is likely to be quite distinct.

\section{References}

Booth, R.S., Johansson, L.E. (1990), these proceedings.

Cohen, R., Dame, T., Garay, G., Montani, J., Rubio, M., Thaddeus, P. (1988), Ap. J. 331, L95.

Dragovan, M., Stark, A. A., Pernic, R., Pomerantz, M. (1990), Appl. Optics 29, 463.

Pajot, F., Gispert, R., Lamarre, J.M., Puget, J. L., Pomerantz, M. A., Peyturaux, R. (1989), Astrophysics in Antarctica, D.J. Mullan, M.A. Pomerantz, T. Stanev (eds.) (AIP Press: New York) p.93.

Schieder, R., Tolls, V., Winnewisser, G. (1989), Experimental Astronomy 1, 101. 